\title{
SOME PROPERTIES OF ANNULAR BLANKETS
}

\author{
C. A. HAYES, JR. AND A. P. MORSE
}

1. Introduction. Given a metric space $S$ and a set $A \subset S$, suppose that there is a function $F$ which correlates to each point $x$ in $A$ such a family of nonvacuous sets that there are members of the family of diameter less than any preassigned positive number, shrinking down upon, but not necessarily containing, the point $x$. Such a function is called a blanket ${ }^{1}$ with domain $A$. It is known that if a blanket possesses suitable covering properties, then almost everywhere differentiation is possible with respect to it. ${ }^{2}$

We deal here with a special type of blanket, which we term an annular blanket. We are interested in learning what effect the ability to differentiate with respect to these annular blankets has upon their covering properties. In this respect, we supplement two earlier papers by A. P. Morse. ${ }^{3}$ In $\$ 4$, we give necessary and sufficient conditions that an annular blanket in a separable space be strong with respect to a given measure. In $\S 5$, we restrict ourselves to the euclidean plane and utilize the properties of a special plane $\operatorname{set}^{4} \mathcal{A}$ together with the results of $\$ 4$, to construct a blanket ${ }^{5}$ whose member sets are closed, nearly circular, and star-shaped about a central point, with respect to which differentiation is impossible for a certain measure $\Omega$. The measure $\Omega$ is obtained by constructing a certain plane set $B_{0}$ and defining $\Omega(\beta)=L\left(B_{0} \beta\right)$, where $L$ denotes Carathéodory linear measure in the plane.

A theorem of A. S. Besicovitch ${ }^{6}$ insures that any blanket, which correlates with a point the closed circular disks concentric about it, may be employed for purposes of differentiation for a wide class of measures, which includes $\Omega$ above. This result has been shown in

Received by the editors June 18, 1948 and, in revised form, October 30, 1948.

${ }^{1}$ Unfamiliar technical terms are defined in $\$ 2$ and $\$ 5$.

${ }^{2}$ See A. P. Morse, $A$ theory of covering and differentiation, Trans. Amer. Math. Soc. vol. 55 (1944) pp. 205-235. Hereinafter this will be referred to as M1.

${ }^{3}$ See M1, and in addition, A. P. Morse, Perfect blankets, Trans. Amer. Math. Soc. vol. 61 (1947) pp. 418-442. Hereinaf ter this will be referred to as M2.

4 See A. P. Morse and J. F. Randolph, The $\phi$ rectifiable subsets of the plane, Trans. Amer. Math. Soc. vol. 55 (1944) pp. 236-305. The set $\mathcal{A}$ is defined and some of its properties are given in pp. 285-286 of that paper. Hereinafter, that paper will be referred to as RM.

${ }^{5}$ Reference to such a blanket is made in pp. 431-432 of M2.

${ }^{6} \mathrm{~A}$. S. Besicovitch, $A$ general form of the covering principle and relative differentiation of additive functions, Proc. Cambridge Philos. Soc. vol. 41 (1945) pp. 103-110. 
M2 to hold for a class of blankets known as star blankets, which includes the circular blankets just mentioned, and also certain types of nearly circular star-shaped blankets, among others. However, our results prove that not all kinds of nearly circular star-shaped blankets are suitable for differentiation.

2. Preliminaries. We now set forth certain notations, conventions, definitions, and other pertinent information which we shall have occasion to use.

Let us agree that $(x \in A)=(x$ is a member of $A)=(x$ is in $A)$; $(x \notin A)=(x$ is not a member of $A)=(x$ is not in $A) ;(A \subset B)=(A$ is a subset of $B)=(B \supset A)=(B$ contains $A)$; the integer 0 and the empty set are identical.

If $a_{x}$ is a set for each $x \in B$ then

$$
\sum_{x \in B} a_{x}=\underset{y}{E}\left[y \in a_{x} \text { for some } x \in B\right] .
$$

If $\mathfrak{F}$ is a family, then

$$
\sigma \mathfrak{F}=\sum_{\beta \in \mathfrak{F}} \beta .
$$

Thus $x \in \sigma \mathfrak{F}$ if and only if $x$ is a member of some set belonging to $\mathfrak{F}$.

If $a_{x} \geqq 0$ for $x$ in a countable set $B$, then

$$
\sum_{x \in B} a_{x}
$$

is also the appropriate numerical sum. Whether numerical summation or set theoretic summation is intended in a particular instance will be clear from the context.

2.1. Definition.

$$
\operatorname{sng} x=\underset{y}{E}[y=x] .
$$

Thus sng $x$ is the set whose sole member is $x$.

We fix throughout this paper a separable metric space $\mathcal{S}$ metrized with $\rho$. Hereafter such words as closed, open, Borel, and so on, will refer to the metric $\rho$.

2.2. Definition.

$$
\mathfrak{c}(x, r)=\underset{y}{E}[\rho(x, y) \leqq r] .
$$

$\complement(x, r)$ is the closed sphere with center $x$ and radius $r$ whenever $x \in \mathcal{S}$ and $r \geqq 0$.

2.3. Definition. For $A \subset S$ we define 


$$
\begin{array}{ll}
\operatorname{diam} A=\sup _{(x \in \Lambda)(\nu \in \Lambda)} \rho(x, y) & \text { whenever } A \neq 0 ; \\
\operatorname{diam} A=0 & \text { whenever } A=0 .
\end{array}
$$

2.4. Definition. For $A \subset S$ we define

$$
\operatorname{nlrg} A=\underset{y}{E}[\rho(x, y) \leqq 2 \operatorname{diam} A \text { for some } x \text { in } A] \text {. }
$$

2.5. Definition. $\phi$ measures $S$ if and only if $\phi$ is such a function on $E_{\beta}[\beta C S]$ to $E_{t}[0 \leqq t \leqq \infty]$ that

$$
\phi(A) \leqq \sum_{\beta \in \mathscr{F}} \phi(\beta)
$$

whenever $\mathfrak{F}$ is a countable family for which $A \subset \sigma \mathfrak{F} \subset S$. Bearing in mind that empty sums are zero, it follows that if $\phi$ measures $S$ then:

I. $\phi(0)=0$;

II. $\phi(B) \leqq \phi(A)$ whenever $B C A C S$;

III. $\phi(\sigma \mathfrak{F}) \leqq \sum_{\beta \in \mathfrak{F}} \phi(\beta)$ whenever $\mathfrak{F}$ is a countable family for which $\sigma \mathfrak{F} \subset S$.

2.6. Definition. The family $\mathfrak{U}$ is defined by: $\phi \in \mathfrak{U}$ if and only if $\phi$ so measures $\mathcal{S}$ that

$$
\phi(A+B)=\phi(A)+\phi(B) .
$$

whenever $\inf _{(x \in A)(y \in B)} \rho(x, y)>0$.

2.7. Definition.

$$
U=\mathfrak{U} \cdot \underset{\phi}{E}[\phi(\beta)<\infty \text { for each bounded set } \beta \subset \mathcal{S}] \text {. }
$$

2.8. Definition. $A$ is $\phi$-measurable if and only if $\phi$ so measures $S$ that

$$
\phi(T)=\phi(T A)+\phi(T-A)
$$

for each subset $T$ of $S$.

2.9. Definition. The limit notations

$$
\operatorname{limalinf}_{\mathfrak{F} \ni \beta \rightarrow x} f(\beta), \quad \operatorname{limalsup}_{\mathfrak{F} \ni \beta \rightarrow x} f(\beta)
$$

are defined, respectively, as

$$
\lim _{t \rightarrow 0+} \inf _{\beta \in H(t)} f(\beta), \quad \lim _{t \rightarrow 0+} \sup _{\beta \in H(t)} f(\beta),
$$

where

$$
H(t)=\mathfrak{F} \cdot \underset{\beta}{E}[\operatorname{diam}(\beta+\operatorname{sng} x)<t] .
$$


We agree that

$$
\operatorname{limal}_{\mathfrak{F} \ni \beta \rightarrow x} f(\beta)=\operatorname{limalsup}_{\mathfrak{F} \ni \beta \rightarrow x} f(\beta)
$$

if and only if

$$
\operatorname{limalsup}_{\mathfrak{F} \ni \beta \rightarrow x} f(\beta)=\operatorname{limalinf}_{\mathfrak{F} \ni \beta \rightarrow x} f(\beta) .
$$

2.10. Definition. We say $F$ is a blanket if and only if $F$ is such a function that for $x$ in its domain

(i) $x \in \mathcal{S}$ and $F(x)$ is a family of non-vacuous subsets of $S$;

(ii) $\beta \in F(x)$ implies diam $\beta<\infty$;

(iii) $\inf _{\beta \in \boldsymbol{r}(x)}$ diam $(\beta+$ sng $x)=0$.

2.11. Definition. If $F$ is a blanket with domain $A$ then the family

$$
\sum_{x \in A} F(x)
$$

is called the spread of $F$.

2.12. Definition. $F$ is a natural blanket if and only if $F$ is a blanket and $x \in \beta$ whenever $\beta \in F(x)$.

2.13. Definition. $F$ is a $\phi$-diametrically regular blanket if and only if $\phi \in U$ and $F$ is such a natural blanket that

$$
\underset{P(x) \ni \beta \rightarrow z}{\operatorname{limalsup}} \frac{\phi(\operatorname{nlrg} \beta)}{\phi(\beta)}<\infty
$$

for $\phi$ almost all $x$ in the domain of $F$.

2.14. Definition. $F$ is a Borelian blanket if and only if $F$ is such a blanket that $\beta$ is a Borel set whenever $\beta \in F(x)$.

2.15. Definition. $F$ is a close blanket if and only if $F$ is such a blanket that $\beta$ is a closed set whenever $\beta \in F(x)$.

2.16. Definition. $F$ is a $\phi$-heavy blanket if and only if $F$ is a Borelian blanket, $\phi \in V$, and the spread of $F$ contains a countable disjointed family which covers $\phi$ almost all of the domain of $F$.

2.17. Definition. $G$ is a subblanket of $F$ if and only if $F$ and $G$ are such blankets that $G(x) \subset F(x)$ whenever $x$ is in the domain of $G$.

2.18. Definition. $F$ is a $\phi$-strong blanket if and only if $F$ is such a blanket that each of its subblankets is $\phi$-heavy.

2.19. Definition. $F$ is a perfect blanket if and only if $F$ is $\phi$-strong for each $\phi \in U$.

2.20. Definition.

$$
D(f, \phi, F, x)=\operatorname{limal}_{\boldsymbol{F}(x) \ni \beta \rightarrow x} \frac{f(\beta)}{\phi(\beta)} .
$$


The next theorem is a consequence of 6.11 of M1 and the one immediately following it is quoted directly from 8.11 of M1.

2.21. TheoRem. Every $\phi$-diametrically regular close blanket is $\phi$ strong.

2.22. Theorem. If $F$ is $\phi$-strong and $f \in U$, then $0 \leqq D(f, \phi, F, x)<\infty$ for $\phi$ almost all $x$ in the domain of $F$.

2.23. Definition. $F$ is a universal blanket if and only if $F$ is such a blanket that whenever $f \in \mathcal{U}$ and $\phi \in \mathcal{U}$, then $0 \leqq D(f, \phi, F, x)<\infty$ for $\phi$ almost all $x$ in the domain of $F$.

A perfect blanket is $\mathbf{2 . 2 2}$ evidently a universal blanket.

2.24. Definition. $F$ is $\phi$-free if and only if $\phi \in V$ and $F$ is such a blanket with domain $A$ that for each Borel set $B C S$,

$$
\operatorname{limal}_{\boldsymbol{r}(\boldsymbol{x}) \exists \beta \rightarrow \boldsymbol{\beta}} \frac{\phi(B \beta)}{\phi(\beta)}=0
$$

for $\phi$ almost all $x \in A \cdot(S-B)$.

2.25. Definition. $F$ is an annular blanket if and only if $F$ is a blanket, and there exists such a function $\lambda$ that for each $x$ in the domain of $F$ :

(i) $1<\lambda(x)<\infty$;

(ii) $F(x)$ is that family of sets for which $\beta \in F(x)$ if and only if $\beta$ is closed and

$$
\mathfrak{S}(x, r) \subset \beta \subset \mathcal{C}(x, r \lambda(x))
$$

for some $r>0$.

2.26. Definition.

$$
\mathfrak{P}_{\phi}=\underset{x}{E}[\phi(\operatorname{sng} x)>0] .
$$

Evidently $\mathfrak{M} \phi$ is a countable set of points in $S$ for each $\phi \in V$.

2.27. Definition.

$$
\Im \phi=\underset{x}{E}\left[\limsup _{r \rightarrow 0+} \frac{\phi\{\Subset(x, 2 r)\}}{\phi\{\mathbb{C}(x, r)\}}=\infty\right] .
$$

2.28. Lemma. If $\phi \in \mathcal{V}, \mu>1$, and

$$
\underset{r \rightarrow 0+}{\limsup } \frac{\phi\{\mathfrak{C}(x, \mu r)\}}{\phi\{\mathfrak{C}(x, r)\}}=\infty
$$

then 


$$
\underset{r \rightarrow 0+}{\limsup } \frac{\phi\{(\mathcal{C}(x, \nu r)\}}{\phi\{\mathfrak{C}(x, r)\}}=\infty
$$

for each $\nu>1$.

Proof. Suppose, on the contrary, $\nu>1$ and $L<\infty$ such that

$$
\underset{r \rightarrow 0+}{\limsup } \frac{\phi\{\mathfrak{G}(x, \nu r)\}}{\phi\{\mathbb{S}(x, r)\}}<L .
$$

It follows by an inductive process that for each positive integer $n$,

$$
\limsup _{r \rightarrow 0+} \frac{\phi\left\{\mathfrak{C}\left(x, \nu^{n} r\right)\right\}}{\phi\{(\mathfrak{C}(x, r)\}}<L^{n} \text {. }
$$

However, we may choose such a positive integer $N$ that $\mu<\nu^{N}$, and our hypotheses imply that

$$
\limsup _{r \rightarrow 0+} \frac{\phi\left\{\mathfrak{S}\left(x, \nu^{N} r\right)\right\}}{\phi\{\mathfrak{S}(x, r)\}} \geqq \underset{r \rightarrow 0+}{\limsup } \frac{\phi\{\mathfrak{S}(x, \mu r)\}}{\phi\{\mathfrak{S}(x, r)\}}=\infty
$$

which contradicts (1) above. The lemma is therefore proved.

We note in passing the following obvious corollary.

2.29. Corollary. If $\phi \in V$ and $\mu>1$, then

$$
\Im \phi=\underset{x}{E}\left[\limsup _{r \rightarrow 0+} \frac{\phi\{(\mathfrak{C}(x, \mu r)\}}{\phi\{\mathfrak{S}(x, r)\}}=\infty\right] .
$$

3. Some preliminary lemmas. For the entirety of $\$ 3$ we choose such a fixed $\phi$ that $\phi \in \mathcal{V}, \mathfrak{M} \phi=0$, and $\phi(S)<\infty$. We shall let $Q=\Im \phi$ for brevity.

3.1. LemMA. If $A$ is a Borel subset of $\mathcal{S}$, and $0 \leqq a \leqq 2 a \leqq \phi(A)$, then there exists $a$ Borel set $B C A$ for which $a \leqq \phi(B) \leqq 2 a$.

Proof. In case $a=0$ the lemma is obviously true. Suppose then that $0<a<\infty$. With the aid of Lindelöf's covering theorem for separable metric spaces, we observe that there exists, and we determine, a countable disjointed family of Borel sets $\beta_{1}, \beta_{2}, \beta_{3}, \cdots$ for which

$$
\sum_{n=1}^{\infty} \beta_{n}=A ; \quad 0 \leqq \phi\left(\beta_{n}\right) \leqq a
$$

for $n=1,2,3, \cdots$.

Since 


$$
\lim _{n \rightarrow \infty} \phi\left(\sum_{m=1}^{n} \beta_{m}\right)=\phi\left(\sum_{m=1}^{\infty} \beta_{m}\right)=\phi(A) \geqq 2 a>0
$$

and also

$$
\phi\left(\sum_{m=1}^{n} \beta_{m}\right)=\sum_{m=1}^{n} \phi\left(\beta_{m}\right)
$$

for $n=1,2,3, \cdots$, it follows that for a suitably chosen positive integer $N$,

$$
a \leqq \phi\left(\sum_{m=1}^{N} \beta_{m}\right) \leqq 2 a .
$$

Setting $B=\sum_{m=1}^{N} \beta_{m}$, the truth of the lemma is apparent.

3.2. Lemma. If $A$ is a Borel subset of $\mathcal{S}$, and $0 \leqq k \leqq \phi(A)$, then there exists a Borel set $C \subset A$ for which $\phi(C)=k$.

Proof. With the aid Lemma 3.1 we may and do determine inductively such a disjointed sequence of Borel sets $0=B_{1}, B_{2}, B_{3}, \cdots$ that for each positive integer $n$,

$$
\begin{gathered}
B_{n+1} \subset\left(A-\sum_{m=1}^{n} B_{m}\right) ; \\
\frac{1}{2}\left\{k-\phi\left(\sum_{m=1}^{n} B_{m}\right)\right\} \leqq \phi\left(B_{n+1}\right) \leqq k-\phi\left(\sum_{m=1}^{n} B_{m}\right) .
\end{gathered}
$$

Let $C=\sum_{m=1}^{\infty} B_{m}$. It is easily checked that $C \subset A$ is a Borel set for which $\phi(C)=k$. The proof of the lemma is complete.

3.3. Definition. $J(B, a, b, \mu, \alpha)$ is the set of those points $x$ such that

$$
\phi\{B \cdot \Subset(x, \mu r)\}>\alpha \phi\{\Subset(x, r)\}
$$

for some $r$ for which $a<r<b$.

3.4. Lemma. If $B \subset S, 1<\mu<\infty, 0<\alpha<\infty, 0 \leqq a<b<\infty$, then $J(B, a, b, \mu, \alpha)$ is open.

Proof. We choose any point $x$ in $J(B, a, b, \mu, \alpha)$ and 3.3 so determine $r$ that

$$
\phi\{B \cdot \Subset(x, \mu r)\}>\alpha \phi\{\Subset(x, r)\} ; \quad a<r<b .
$$

Since $\phi \in V$ we determine $d>0$ so that

$$
\phi\{B \cdot \mathbb{C}(x, \mu r)\}>\alpha \phi\{\Subset(x, r+d)\} ; \quad a<r+d<b .
$$


Now let $y$ be any point in $(\mathcal{S}(x, d / 2)$. It is easily checked that

$$
\begin{aligned}
& \mathfrak{S}(x, r) \subset \mathfrak{C}(y, r+d / 2) \subset \mathfrak{C}(x, r+d) ; \\
& \mathfrak{S}(x, \mu r) \subset \mathfrak{C}(y, \mu(r+d / 2)) .
\end{aligned}
$$

From (1) and (2) we note that

$$
\phi\{B \cdot \mathbb{E}(y, \mu(r+d / 2))\}>\alpha \phi\{\mathbb{E}(y, r+d / 2)\} ; \quad a<r+d / 2<b .
$$

Accordingly $y \in J(B, a, b, \mu, \alpha)$.

The last paragraph assures us that $\mathbb{C}(x, d / 2) \subset J(B, a, b, \mu, \alpha)$, and hence from the arbitrary nature of $x$ in $J(B, a, b, \mu, \alpha)$, we see that $J(B, a, b, \mu, \alpha)$ is open. The lemma is proved.

3.5. Lemma. If $\epsilon>0,1<\mu<\infty, 0<\alpha<\infty, 0<b<\infty$, then there is a positive number a for which

$$
\phi\{Q-J(S, a, b, \mu, \alpha)\}<\epsilon ; \quad a<b ; a<\epsilon .
$$

Proof. From Corollary 2.29 we note that $Q \subset J(S, 0, b, \mu, \alpha)$. The desired result follows at once with the aid of Lemma 3.4.

3.6. Lemma. If $\epsilon>0,1<\mu<\infty, 1 \leqq \alpha<\infty, 0<b<\infty$, then there is a positive number $a$ and $a$ Borel set $B$ such that

$$
\begin{gathered}
\alpha \phi(B)=\phi(S) ; \\
\phi\left\{Q-J\left(B, a, b, \mu^{2}, 1\right)\right\}<\epsilon ; \quad a<b ; a<\epsilon .
\end{gathered}
$$

Proof. In accordance with Lemma 3.5 , we choose such a positive number $a$ that

$$
\phi\{Q-J(S, a, b, \mu, \alpha)\}<\epsilon ; \quad a<b ; a<\epsilon .
$$

Using the fact that $S$ is a separable space we construct such a countable disjointed family $T$ of Borel sets that $\sigma T=S$ and

$$
\operatorname{diam} t<\mu(\mu-1) a
$$

whenever $t \in T$. We invoke Lemma 3.2 to construct such a countable disjointed family $S$ of Borel sets in one-to-one correspondence with $T$ that

$$
\phi(s)=\frac{1}{\alpha} \phi(t) ; \quad s \subset t
$$

whenever $s \in S$ corresponds to $t \in T$.

We let

$$
B=\sigma S
$$


we see that $B$ is a Borel set and

$$
\phi(B)=\sum_{, \in S} \phi(s)=\frac{1}{\alpha} \sum_{t \in T} \phi(t)=\frac{1}{\alpha} \phi(\sigma T)=\frac{1}{\alpha} \phi(S) .
$$

We let $x$ be any point in $J(S, a, b, \mu, \alpha)$. We 3.3 choose a number $r$ so that

$$
\phi\{\mathbb{C}(x, \mu r)\}>\alpha \phi\{\mathfrak{C}(x, r)\}, \quad a<r<b,
$$

let

$$
\begin{aligned}
M & =\underset{t}{E}[t \in T \text { and } t \cdot \mathbb{E}(x, \mu r) \neq 0], \\
N & =\underset{E}{E}[s \in S \text { and } s \text { corresponds to some } t \in M \text { in the sense of (3)], }
\end{aligned}
$$

and observe that $M$ and $N$ are countable disjointed families of Borel sets. From (2), (3), and (4) we obtain

$$
\begin{gathered}
\mathfrak{C}(x, \mu r) \subset \sigma M ; \quad \sigma N \subset \mathbb{C}\left(x, \mu^{2} r\right) ; \\
\phi\left\{B \cdot \mathbb{C}\left(x, \mu^{2} r\right)\right\} \geqq \phi(\sigma N)=\sum_{s \in N} \phi(s)=\frac{1}{\alpha} \sum_{t \in M} \phi(t)=\frac{1}{\alpha} \phi(\sigma M) \\
\geqq \frac{1}{\alpha} \phi\{\mathbb{C}(x, \mu r)\}>\phi\{\mathbb{C}(x, r)\} .
\end{gathered}
$$

Consequently $x \in J\left(B, a, b, \mu^{2}, 1\right)$.

The last paragraph assures us that

$$
J(S, a, b, \mu, \alpha) \subset J\left(B, a, b, \mu^{2}, 1\right),
$$

and a glance at (1) now convinces us that

$$
\phi\left\{Q-J\left(B, a, b, \mu^{2}, 1\right)\right\}<\epsilon ; \quad a<b ; a<\epsilon .
$$

The proof is complete.

3.7. LEMMA. If $F$ is any $\phi$-free annular blanket with domain $A$, then $\phi(Q \cdot A)=0$.

Proof. We shall prove the lemma by contradiction. We let $Q^{\prime}=Q A$ and suppose that $\phi\left(Q^{\prime}\right)>0$. We choose any sequence of numbers $\infty .>\mu_{1}>\mu_{2}>\mu_{3}>\cdots$ for which $\lim _{k \rightarrow \infty} \mu_{k}=1$. In accordance with Lemma 3.6 we determine inductively such a sequence of positive numbers $1=a_{1}>a_{2}>a_{3}>\cdots$ that $\lim _{k \rightarrow \infty} a_{k}=0$, and a sequence of Borel sets $B_{1}, B_{2}, B_{3}, \cdots$ for which

$$
\phi\left(B_{k}\right) \leqq \frac{\phi\left(Q^{\prime}\right)}{2^{k+1}} ; \quad \phi\left\{Q-J\left(B_{k}, a_{k+1}, a_{k}, \mu_{k}^{2}, 1\right)\right\}<\frac{\phi\left(Q^{\prime}\right)}{2^{k+1}}
$$


for $k=1,2,3, \cdots$. We let

$$
C=A \cdot \prod_{k=1}^{\infty} J\left(B_{k}, a_{k+1}, a_{k}, \mu_{k}^{2}, 1\right),
$$

and note from (1) that

$$
\begin{aligned}
\phi\left(Q^{\prime}-C\right) & \leqq \phi\left\{Q-\prod_{k=1}^{\infty} J\left(B_{k}, a_{k+1}, a_{k}, \mu_{k}^{2}, 1\right)\right\} \\
& <\sum_{k=1}^{\infty} \frac{\phi\left(Q^{\prime}\right)}{2^{k+1}}=\frac{\phi\left(Q^{\prime}\right)}{2} ; \phi(C)>\frac{\phi\left(Q^{\prime}\right)}{2} .
\end{aligned}
$$

We let

$$
B=\sum_{k=1}^{\infty} B_{k},
$$

note that $B$ is a Borel set, perceive from (1) above that

$$
\phi(B) \leqq \sum_{k=1}^{\infty} \phi\left(B_{k}\right) \leqq \sum_{k=1}^{\infty} \frac{\phi\left(Q^{\prime}\right)}{2^{k+1}}=\frac{\phi\left(Q^{\prime}\right)}{2},
$$

and observe from (2) and (3) that

$$
\phi(C-B)>0 .
$$

Next, we choose any point $x$ in $C-B$ and any positive integer $k$. Since $x \in C$, we 3.3 determine such a positive number $r$ that

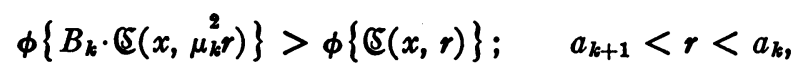

select such a closed set $\alpha$ that $^{7}$

$$
\phi(\alpha)>(1 / 2) \phi\left\{B_{k} \cdot \Subset\left(x, \underset{\mu_{k} r}{2}\right)\right\} ; \quad \alpha \subset B_{k} \cdot \mathbb{C}\left(x, \mu_{k}^{2} r\right),
$$

and let $\beta=\mathfrak{c}(x, r)+\alpha$. Clearly $\beta$ is closed. From (6) we note that

$$
\mathfrak{S}(x, r) \subset \beta \subset \mathfrak{S}\left(x, \stackrel{2}{\mu_{k} r}\right) \text {, }
$$

and from (5) and (6) we derive

$$
\frac{\phi(B \beta)}{\phi(\beta)} \geqq \frac{\phi\left(B_{k} \beta\right)}{\phi(\beta)} \geqq \frac{\phi(\alpha)}{\phi\{\mathfrak{夭}(x, r)\}+\phi(\alpha)}>\frac{\phi(\alpha)}{3 \phi(\alpha)}=\frac{1}{3} .
$$

${ }^{7}$ See A. P. Morse, Role of internal families in measure theory, Bull. Amer. Math. Soc. vol. 50 (1944) pp. 723-728. 
It is clear that $(C-B) \subset A$, and from $2.25,(7),(8)$, and the arbitrary nature of $x$ in the last paragraph, it follows that

$$
\operatorname{limalsup}_{\boldsymbol{F}(x) \ni \beta \rightarrow x} \frac{\phi(B \beta)}{\phi(\beta)}>0
$$

for each $x$ in $C-B$. Due to (4), it is 2.24 clear that $F$ is not $\phi$-free. This contradiction of our hypotheses proves the lemma.

\section{Necessary and sufficient conditions that an annular blanket be $\psi$-strong.}

4.1. THEOREM. If $F$ is a $\psi$-free annular blanket with domain $A$, then $A \cdot \Im \psi$ is of $\psi$-measure zero.

Proof. It is not difficult to see that if the theorem holds when $\psi(\delta)<\infty$, it also holds when $\psi(\delta)=\infty$. We therefore prove the theorem under the assumption that $\psi(S)<\infty$.

We recall 2.26 , and represent $\psi$ as the sum of two measures $\theta$ and $\phi$ defined by

$$
\theta(\beta)=\psi(\beta \cdot \mathfrak{M} \psi) ; \quad \phi(\beta)=\psi(\beta)-\theta(\beta) .
$$

Since $\mathfrak{M} \psi$ is a countable set and $F$ is $2.24 \psi$-free by hypothesis, it is clear that

$$
\operatorname{limal}_{\boldsymbol{P}(x) \ni \beta \rightarrow x} \frac{\theta(\beta)}{\psi(\beta)}=\operatorname{limal}_{\boldsymbol{F}(x) \ni \beta \rightarrow x}\left\{\frac{\theta(\beta)}{\phi(\beta)+\theta(\beta)}\right\}=0
$$

for $\psi$-almost all $x$ in $A \cdot(S-\mathfrak{M} \psi)$; from this it follows that

$$
\operatorname{limal}_{\boldsymbol{r}(x) \ni \beta \rightarrow x} \frac{\theta(\beta)}{\phi(\beta)}=0
$$

for $\psi$ almost all $x$ in $A \cdot(S-\mathfrak{M} \psi)$, and hence from (1), for $\phi$ almost all $x \in A$.

We choose any Borel set $B C S$. From (2) and the fact that $F$ is $\psi$-free by hypothesis, we derive

$$
\operatorname{limal}_{\boldsymbol{r}(x) \ni \beta \rightarrow x} \frac{\phi(B \beta)}{\phi(\beta)} \leqq \operatorname{limal}_{\boldsymbol{P}(x) \ni \beta \rightarrow x} \frac{\psi(B \beta)}{\psi(\beta)} \cdot \operatorname{limal}_{\boldsymbol{r}(x) \ni \beta \rightarrow x}\left\{1+\frac{\theta(\beta)}{\phi(\beta)}\right\}=0
$$

for $\phi$ almost all $x$ in $A \cdot(S-B)$. Hence $F$ is $\phi$-free. From (1) we see that $\phi \in \mathcal{V}, \mathfrak{M} \phi=0$, and $\phi(S)<\infty$; we may therefore apply Lemma 3.7 to conclude that

$$
\phi(A \cdot \Im \phi)=0 .
$$

From (2) and (3) we derive 


$$
\begin{aligned}
\limsup _{r \rightarrow 0+} \frac{\psi\{\mathfrak{C}(x, 2 r)\}}{\psi\{\mathfrak{C}(x, r)\}} & \leqq \limsup _{r \rightarrow 0+} \frac{\psi\{\mathfrak{C}(x, 2 r)\}}{\phi\{\mathfrak{C}(x, r)\}} \\
& =\limsup _{r \rightarrow 0+} \frac{\phi\{\mathfrak{C}(x, 2 r)\}}{\phi\{\mathfrak{C}(x, r)\}} \cdot\left\{1+\frac{\theta\{\mathfrak{C}(x, 2 r)\}}{\phi\{\mathfrak{C}(x, 2 r)\}}\right\} \\
& =\limsup _{r \rightarrow 0+} \frac{\phi\{\mathfrak{C}(x, 2 r)\}}{\phi\{\mathfrak{C}(x, r)\}}<\infty
\end{aligned}
$$

for $\phi$ almost all $x$ in $A$. It is clear that

$$
\underset{r \rightarrow 0+}{\limsup } \frac{\psi\{\mathfrak{\complement}(x, 2 r)\}}{\psi\{\mathfrak{S}(x, r)\}}<\infty
$$

for each $x \in \mathfrak{M} \psi$. It is therefore apparent that (4) holds for $\psi$ almost all $x \in A$. Thus we 2.27 observe that $A \cdot \mathfrak{F} \psi$ is of $\psi$-measure zero. The theorem is proved.

4.2. TheOREM. If $F$ is a $\psi$-free annular blanket, then $F$ is $\psi$-diametrically regular.

Proof. The desired conclusion follows from 4.1, 2.25, 2.29, and reference to Definition 2.13.

4.3. Theorem. $A$ necessary and sufficient condition that an annular blanket $F$ be $\psi$-strong is that it be $\psi$-free.

Proof. We check the sufficiency first. If $F$ is $\psi$-free it is $4.2 \psi$-diametrically regular, and hence is $2.21 \psi$-strong.

We now prove the necessity by contradiction. We suppose $F$ to be $\psi$-strong yet not $\psi$-free. With the aid of 2.20 and 2.22 we determine a bounded Borel set $B$, a bounded set $D C(S-B)$ of positive $\psi$-measure, and a number $d$ for which

$$
\operatorname{limal}_{\boldsymbol{r}(x) \ni \beta \rightarrow x} \frac{\psi(B \beta)}{\psi(\beta)}>d>0
$$

whenever $x \in D$.

We select such a closed set $C \subset B$ that ${ }^{8}$

$$
\psi(B-C)<d \psi(D)
$$

choose a bounded open set $S$ containing $B+D$, let

$$
G(x)=F(x) \cdot \underset{\beta}{E}\left[\beta \subset(S-C) ; \frac{\psi(B \beta)}{\psi(\beta)}>d\right],
$$

\footnotetext{
${ }^{8}$ See footnote 7.
} 
for each $x \in D$, and observe that $G$ is a subblanket of $F$ whose domain is $D$. Thus $G$ is $2.18 \psi$-heavy. We 2.16 extract from the spread of $G$ such a countable disjointed family $\tau$ that

$$
\psi(D-\sigma \tau)=0,
$$

and from (2) and (3) observe that

$$
\begin{aligned}
0<\psi(D) \leqq \psi(\sigma \tau) & =\sum_{\beta \in \tau} \psi(\beta)<\frac{1}{d} \sum_{\beta \in \tau} \psi(B \beta) \\
& =\frac{1}{d} \psi(B \sigma \tau) \leqq \frac{1}{d} \psi(B-C) .
\end{aligned}
$$

This last result contradicts (1) and proves the theorem.

5. A nearly spherical, star-shaped blanket which is not universal. In this section we shall construct the example mentioned in the introduction. For the remainder of the paper we shall let $S$ be the euclidean plane, $\rho$ the usual metric. We shall let $L$ denote Carathéodory linear measure in $S$.

We now proceed to define a special set ${ }^{9}$ in $S$ which plays a basic role in the ensuing work. We shall hereinafter call a set in $\mathbf{S}$ a triangle if and only if it is a closed set of points consisting of the interior and boundary of an equilateral triangle with its base line horizontal and below the opposite vertex. Thus the length of each side of a triangle is equal to its diameter.

For $T$ a triangle and $n$ a positive integer, we construct $2 n+1$ triangles, each with diameter equal to

$$
\frac{\operatorname{diam} T}{2 n+1}
$$

so placed that the union of their bases is the base of $T$; next, we take the middle triangle and translate it vertically upward until its top vertex coincides with the top vertex of $T$; we denote the $2 n+1$ triangles so obtained by $O_{n}(T)$. We let $T_{0}$ be a triangle of diameter 1 . We put $\mathfrak{F}_{0}=$ sng $T_{0}$; we so define $\mathfrak{F}_{1}, \mathfrak{F}_{2}, \mathfrak{F}_{3}, \cdots$ that

$$
\mathfrak{F}_{n}=\sum_{T \in \mathfrak{F}_{n-1}} O_{n}(T)
$$

for each positive integer $n$.

We see that $\sigma \mathfrak{F}_{1} \supset \sigma \mathfrak{\mho}_{2} \supset \sigma \mathfrak{F}_{3} \ldots$ and define

$$
\mathfrak{F}=\mathfrak{F}_{1}+\mathfrak{F}_{2}+\mathfrak{F}_{3}+\cdots ;
$$

' See pp. 285-286 of RM. 


$$
\mathcal{A}=\prod_{n=1}^{\infty} \sigma \mathfrak{F}_{n}
$$

Since $T \in \mathcal{F}$ implies that the projection of $A T$ on the base of $T$ is the base of $T$, we have little difficulty in verifying that $L(\mathcal{A T})$ $=\operatorname{diam} T$ for $T \in \mathfrak{F}$. In particular, $L(\mathcal{A})=1$.

We shall term a triangle in $\mathfrak{F}$ an apex triangle if and only if its upper vertex coincides with the top vertex of some larger triangle in $\mathfrak{F}$. We let $W$ be the set so defined that $x \in W$ if and only if $x \in \mathcal{A}$ and the number of apex triangles to which $x$ belongs is finite. Since

$$
\prod_{n=1}^{\infty}\left(1-\frac{1}{2 n+1}\right)=0
$$

we conclude that $L(W)=0$. We let $V=A-W$ and see that $L(V)$ $=L(\mathcal{A})=1$.

If $x$ is any point in $V$, there clearly exists a countable family $\mathbb{B}$ of triangles whose diameters tend to zero, such that whenever $T \in \$$ we can find such a triangle $T^{\prime}$ and such a positive integer $n$ that $T \in O_{n}\left(T^{\prime}\right) ; T$ is an apex triangle; $x \in T$. Let $r=1 / 3$ diam $T^{\prime}$. It is easily seen that $\mathbb{E}(x, r)$ contains $T$, and contains no points of other triangles belonging to $O_{n}\left(T^{\prime}\right) ; \mathfrak{S}(x, 3 r)$ contains the whole triangle $T^{\prime}$. Thus

$$
\frac{L(\mathcal{A} \cdot(\mathfrak{C}(x, 3 r))}{L(\mathcal{A} \cdot(\mathfrak{C}(x, r))}=2 n+1 .
$$

We see from this that

$$
\underset{r \rightarrow 0+}{\limsup } \frac{L(\mathcal{C} \cdot(\mathfrak{C}(x, 3 r))}{L(\mathcal{A} \cdot(\mathfrak{C}(x, r))}=\infty
$$

for each $x \in V$.

Hereinafter we shall let $\phi$ denote that measure for which $\phi(\beta)$ $=L(\mathcal{A} \beta)$ for each $\beta C S$. From the preceding remarks we see that $V \subset \Im \phi$ and thus $\phi(\Im \phi)=1$.

5.1. Definition. We say that a set of points $\beta C S$ is star-shaped about $x$ if and only if $x \in \beta$, and every point of the closed line segment joining $y$ to $x$ belongs to $\beta$ whenever $y \in \beta$.

5.2. Definition. We say that $G$ is a star-shaped blanket if and only if $G$ is such a blanket that all members of $G(x)$ are star-shaped about $x$ whenever $x$ is in the domain of $G$.

5.3. Definition. We define $S$ as that function for which $S(x, \beta)$ is the set of points comprising the union of those straight line seg- 
ments joining $x$ to the points of $\beta$.

Evidently $S(x, \beta)$ is star-shaped about $x$ for each $x \in \mathcal{S}, \beta \subset S$.

5.4. Definition. We term internal radius of $\beta$ at $x$ the sup of numbers $r$ for which $\subseteq(x, r) \subset \beta$.

5.5. Definition. We define $g$ as that function for which

$$
g(r)=\inf _{x \in \mathcal{C} A} \phi\{\Subset(x, r)\} .
$$

5.6. LEMMA. $g(r)>0$ whenever $r>0$.

This is easily verified by routine methods.

5.7. Lemma. For each $\epsilon>0$ there exists such a number $r>0$ that $\phi\{S(x, \mathfrak{E}(y, r))\} \leqq \epsilon$ whenever $x \in \mathcal{A}, y \in \mathcal{S}$.

Proof. We choose $\epsilon>0$. It is easily seen that $\phi\{\mathfrak{C}(x, \epsilon / 2)\}$ $=L\{\mathcal{A} \cdot \mathfrak{\complement}(x, \epsilon / 2)\} \leqq \operatorname{diam}(\mathcal{E}(x, \epsilon / 2)=\epsilon$ for each $x \in \mathcal{S}$. It is also known ${ }^{10}$ that any straight line in the plane may intersect $\mathcal{A}$ in at most a set of $\phi$-measure zero. Due to this, we may and do determine such a number $b>0$ that each circular sector of central angle less than $b$, whose center is a point of $\mathcal{A}$, is of $\phi$-measure less than $\epsilon$.

We further determine such a number $r>0$ that for each $x \in S$, $y \in S$,

(i) $\mathcal{S}\{x, \mathfrak{S}(y, r)\}$ is contained in a sector of central angle less than $b$ whenever $\rho(x, y) \geqq \epsilon / 4$;

(ii) $\mathcal{S}\{x, \mathcal{S}(y, r)\} \subset \mathbb{S}(x, \epsilon / 2)$ whenever $\rho(x, y)<\epsilon / 4$.

From the preceding two paragraphs we conclude that $\phi\{S(x$, $\mathfrak{S}(y, r))\} \leqq \epsilon$ whenever $x \in \mathcal{A}, y \in \mathcal{S}$. The proof of the lemma is complete.

We recall Definition 3.3, and prove the following lemma.

5.8. Lemma. If $0<a<b<\infty, 1<\mu<\infty, 0<\alpha<\infty$, then there is such a Borel set $B C S$ that for each $x \in \mathcal{C} A \cdot\{J(S, a, b, \mu, \alpha)-B\}$ there exist some $r$ and $\beta$ for which:

(i) $\beta$ is closed and star-shaped about $x$;

(ii) $\sqrt{ }(x, r) \subset \beta \subset\left(\subseteq\left(x, \mu^{2} r\right) ; a<r<b\right.$;

(iii) $\phi\{\mathbb{E}(x, r)\}<L(B \beta) \leqq L(B)=1 / \alpha$;

(iv) $\phi(\beta)<2 \phi\{\Subset(x, r)\}$;

(v) $\phi(B)=0$.

Proof. We choose such a disjointed family $T$ of squares of equal size, half open to the right and on top, that $\sigma T=S$ and

${ }^{10}$ In fact, if $C$ denotes any strictly rectifiable curve in $\mathrm{S}$, then $L(\mathcal{A} \cdot C)=0$. This may be established by the following references in RM: 2.13, 2.16, 11.1 (1) and (4), and the remark in the middle of page 286 that $A$ is not $L$-rectifiable. 
(1) $\operatorname{diam} t<\mu(\mu-1) a$

whenever $t \in T$; we denote by $M$ the family of all sets $\beta$ of the form $\beta=\mathbb{E}(x, \mu r)$ for which

$$
\phi\{\mathfrak{S}(x, \mu r)\}>\alpha \phi\{\mathfrak{C}(x, r)\} ; \quad a<r<b ;
$$

and we let

$$
T^{\prime}=T \cdot \underset{t}{E}[t \cdot \sigma M \neq 0 ; \phi(t)>0] .
$$

It is clear that $T^{\prime}$ is a finite subset of $T$. We let $N$ denote the number of members of $T^{\prime}$.

For each $t \in T^{\prime}$, we proceed as follows. With the aid of 5.6 and 5.7, we construct a circular disk $\Delta_{t}$ whose center coincides with that of $t$, of radius so small that

$$
\Delta_{t} \subset t ; \quad \phi\left\{S\left(x, \Delta_{t}\right)\right\} \leqq \frac{g(a)}{N+1}
$$

for each $x \in \mathcal{A}$. Next we construct such a countable family $3 \mathfrak{z}$ of concentric circles that

$$
L\left(B_{t}\right)=\frac{1}{\alpha} \phi(t)>0 ; \quad \sigma B_{t} \subset \Delta_{t} ;
$$

and we let

$$
B=\sum_{t \in T^{\prime}} \sigma \mathbb{B}_{t}
$$

Clearly $B$ is a Borel set; it is the totality of points belonging to an enumerable family of circles. For this reason ${ }^{11}$

$$
\phi(B)=L(B \mathcal{A})=0,
$$

which confirms (v) above.

We let $Q^{\prime}=\mathcal{A} \cdot\{J(S, a, b, \mu, \alpha)-B\}$, choose any point $x$ in $Q^{\prime}$, determine such an $r$ that (2) holds, let

$$
T^{\prime \prime}=T^{\prime} \cdot \underset{t}{E}[t \cdot \mathcal{C}(x, \mu r) \neq 0],
$$

and put

$$
\beta=\Subset(x, r)+\sum_{i \in T^{\prime \prime}} S\left(x, \Delta_{\imath}\right) .
$$

Since $T^{\prime \prime}$ has at most finitely many members, it follows that $\beta$ is

"See footnote 10 . 
closed. It is likewise obvious that $\beta$ is star-shaped about $x$. This confirms (i) above.

From (1), (2), and (3) we see that

$$
\Delta_{t} \subset t \subset \mathbb{C}\left(x, \mu^{2} r\right) ; \quad S\left(x, \Delta_{\imath}\right) \subset \mathbb{C}\left(x, \mu^{2} r\right)
$$

for each $t \in T^{\prime \prime}$, and hence

$$
\sum_{t \in T^{\prime \prime}} S\left(x, \Delta_{\imath}\right) \subset \mathfrak{C}\left(x, \mu^{2} r\right) .
$$

We therefore see that

$$
\mathfrak{C}(x, r) \subset \beta \subset \mathfrak{C}\left(x, \mu^{2} r\right),
$$

thereby confirming (ii).

We next observe that

(7) $L(B)=\sum_{\imath \in T^{\prime}} L\left(\sigma 3_{\imath}\right)=\frac{1}{\alpha} \sum_{\imath \in T^{\prime}} \phi(t)=\frac{1}{\alpha} \phi\left(\sigma T^{\prime}\right)=\frac{1}{\alpha} \phi(S)=\frac{1}{\alpha}$,

and that

$$
\begin{aligned}
L(B \beta) & \geqq L\left\{\sum_{t \in T^{\prime \prime}} B \cdot S\left(x, \Delta_{t}\right)\right\} \geqq L\left\{\sum_{t \in T^{\prime \prime}} B \Delta_{t}\right\} \\
& =L\left\{\sum_{t \in T^{\prime \prime}} \sigma B_{t}\right\}=\sum_{t \in T^{\prime \prime}} L\left(\sigma B_{t}\right)=\frac{1}{\alpha} \sum_{t \in T^{\prime \prime}} \phi(t) \\
& =\frac{1}{\alpha} \phi\left(\sigma T^{\prime \prime}\right) .
\end{aligned}
$$

It follows from the definition of $T^{\prime \prime}$ that

$$
\phi\left\{\mathfrak{c}(x, \mu r)-\sigma T^{\prime \prime}\right\}=0 .
$$

Accordingly from (2), (8), and (9) we derive

$$
L(B \beta) \geqq \frac{1}{\alpha} \phi\left(\sigma T^{\prime \prime}\right) \geqq \frac{1}{\alpha} \phi\{\Subset(x, \mu r)\}>\phi\{\Subset(x, r)\},
$$

which, together with (7), completes the confirmation of (iii).

Finally, from (3) and Definition 5.5 we obtain

$$
\begin{aligned}
& \phi(\beta) \leqq \phi\{\Subset(x, r)\}+\sum_{t \in T^{\prime \prime}} \phi\left\{S\left(x, \Delta_{\imath}\right)\right\} \leqq \phi\{\Subset(x, r)\}+\frac{N}{N+1} g(a) \\
& <\phi\{\Subset(x, r)\}+g(a) \leqq 2 \phi\{\Subset(x, r)\},
\end{aligned}
$$

which confirms (iv) and completes the proof of the lemma. 
5.9. THEOREM. There exists a Borel set $B_{0} \subset S, a$ measure $\Omega \in U$, and a close star-shaped blanket $G$, for which

(i) $\Omega(\beta)=L\left(B_{0} \beta\right)$ for each $\beta C S$;

(ii) $G$ is not $\Omega$-free;

(iii) for each $x$ in the domain of $G$

$$
\operatorname{limal}_{G(x) \ni \beta \rightarrow x}(\operatorname{diam} \beta)(\text { internal radius of } \beta \text { at } x)^{-1}=2 .
$$

Proof. We let $Q=\Im \phi$. We select any sequence of numbers $\infty>\mu_{1}$ $>\mu_{2}>\mu_{3}>\cdots$ for which $\lim _{k \rightarrow \infty} \mu_{k}=1$. Since $\phi \in V, \mathfrak{N} \phi=0$, and $\phi(\delta)<\infty$, we may employ Lemma 3.5 to define inductively such a sequence of numbers $1=a_{1}>a_{2}>a_{3}>\cdots$ that $\lim _{k \rightarrow \infty} a_{k}=0$, and for which

$$
\phi\left\{Q-J\left(S, a_{k+1}, a_{k}, \mu_{k}, 2^{k}\right)\right\}<\frac{1}{2^{k+1}}
$$

for $k=1,2,3, \cdots$.

For brevity, we let

$$
D_{k}=J\left(S, a_{k+1}, a_{k}, \mu_{k}, 2^{k}\right)
$$

and

$$
D=\mathcal{A} \cdot \prod_{k=1}^{\infty} D_{k}
$$

Accordingly we observe

$$
\phi\left\{Q-\prod_{k=1}^{\infty} D_{k}\right\} \leqq \sum_{k=1}^{\infty} \phi\left(Q-D_{k}\right) \leqq \sum_{k=1}^{\infty} \frac{1}{2^{k+1}}=\frac{1}{2},
$$

recall that $\phi(Q)=1$, and note that

$$
\begin{aligned}
\phi(D) & =\phi\left(\prod_{k=1}^{\infty} D_{k}\right) \geqq \phi(Q)-\phi\left(Q-\prod_{k=1}^{\infty} D_{k}\right) \\
& \geqq 1-\frac{1}{2}=\frac{1}{2}>0 .
\end{aligned}
$$

With the aid of Lemma 5.8 we construct such a sequence of Borel sets $B_{1}, B_{2}, B_{3}, \cdots$ that for each $x \in \mathcal{A} \cdot\left(D_{k}-B_{k}\right)$ we may find some $r$ and $\beta$ for which

$\beta$ is closed and star-shaped about $x$;

$$
\mathfrak{C}(x, r) \subset \beta \subset\left(\mathcal{E}\left(x, \mu_{k}^{2} r\right) ; \quad a_{k+1}<r<a_{k} ;\right.
$$




$$
\begin{aligned}
& \phi\{\mathfrak{C}(x, r)\}<L\left(B_{k} \beta\right) \leqq L\left(B_{k}\right)=\frac{1}{2^{k}} ; \\
& \phi(\beta)<2 \phi\{\mathfrak{S}(x, r)\} \\
& \phi\left(B_{k}\right)=0 .
\end{aligned}
$$

We let

$$
B=\sum_{k=1}^{\infty} B_{k}
$$

observe that $B$ is a Borel set, note from (6) that $\phi(B)=0$, let $B_{0}=B$ $+\mathcal{A}$, and define

$$
\Omega(\beta)=\phi(\beta)+L(B \beta)=L(\mathcal{A} \beta)+L(B \beta)=L\{(\mathcal{A}+B) \beta\}=L\left(B_{0} \beta\right)
$$

for each $\beta C S$.

From (4) we find

$$
L(B) \leqq \sum_{k=1}^{\infty} L\left(B_{k}\right)=\sum_{k=1}^{\infty} \frac{1}{2^{k}}=1
$$

and hence

$$
\Omega(S)=\phi(S)+L(B) \leqq 2,
$$

which shows that $\Omega \in \mathcal{U}$ and completes the confirmation of (i).

From (1) we see that

$$
\Omega(D-B)=\phi(D-B)=\phi(D) \geqq 1 / 2>0 .
$$

We choose any $x$ in $D-B$ and any positive integer $k$, determine an $r$ and a $\beta$ for which (2), (3), (4), and (5) hold, and observe that

(8) $\frac{\Omega(B \beta)}{\Omega(\beta)} \geqq \frac{L(B \beta)}{\phi(\beta)+L(B \beta)} \geqq \frac{L\left(B_{k} \beta\right)}{\phi(\beta)+L\left(B_{k} \beta\right)}>\frac{L\left(B_{k} \beta\right)}{3 L\left(B_{k} \beta\right)}=\frac{1}{3}>0$.

We may therefore define a close, star-shaped blanket $G$ of domain $D-B$, which, by virtue of (3), satisfies (iii). It is clear from (7) and (8) that $G$ is not $\Omega$-free, which confirms (ii) and completes the proof of the theorem.

5.10. CoRollary. There exists a close, star-shaped blanket $F$ for which

(i) $\operatorname{limal}_{F(x) \ni^{\beta \rightarrow x}}(\operatorname{diam} \beta)$ (internal radius of $\beta$ at $\left.x\right)^{-1}=2$.

(ii) $F$ is not universal.

Proof. We construct $\Omega$ and $G$ as in Theorem 5.9. We further select any blanket $H$ for which domain $H=\operatorname{domain} G$, and $\beta$ is a closed circle 
centered at $x$ whenever $\beta \in H(x)$. We let $F$ be that blanket for which

$$
F(x)=G(x)+H(x)
$$

for each $x \in$ (domain $G$ ). It is clear from (iii) of 5.9 that $F$ is a close star-shaped blanket for which

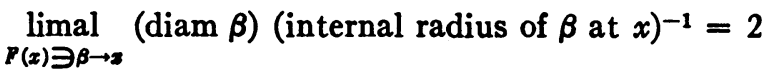

for each $x \in$ (domain $G)$. This confirms (i) above.

It is known ${ }^{12}$ that $H$ is a 2.19 perfect blanket and is therefore $\Omega$-strong. It is easy to see by the method of proof used in Theorem 4.3 that $H$ is $\Omega$-free. However, we know that $G$ is not $\Omega$-free; hence we 2.24 determine a Borel set $B$ and a set $D C(S-B)$ for which $\Omega(D)>0$ and

$$
\operatorname{limal}_{H(x) \ni \beta \rightarrow x} \frac{\Omega(B \beta)}{\Omega(\beta)}=0 ; \quad \operatorname{limal}_{G(x) \exists \beta \rightarrow x} \frac{\Omega(B \beta)}{\Omega(\beta)} \neq 0
$$

for each $x \in D$. It is therefore clear from 2.20 and 2.23 that $F$ is not universal. The proof is complete.

University of California, Davis, Calif. and University of California, Berkeley, Calit.

12 See footnote 6 . 